\title{
MicroRNA-379-5p/YBXI Axis Regulates Cellular EMT to Suppress Migration and Invasion of Nasopharyngeal Carcinoma Cells
}

This article was published in the following Dove Press journal: Cancer Management and Research

\author{
Fei Zhang' \\ Chuanxin Duan' \\ Shucheng Yin ${ }^{2}$ \\ Ying Tian' \\ 'Department of Otolaryngology, \\ Maternal and Child Health Care Hospital \\ of Hubei Province and Women and \\ Children's Hospital of Hubei Province, \\ Wuhan 430070, People's Republic of \\ China; ${ }^{2}$ Department of \\ Otorhinolaryngology-Head and Neck \\ Surgery, Zhongnan Hospital of Wuhan \\ University, Wuhan 43007I, People's \\ Republic of China
}

Background: Epithelial-mesenchymal transition (EMT) is a major actor modulating the metastasis of nasopharyngeal carcinoma (NPC). Increasing evidence indicates that microRNAs (miRs) are the important regulators of EMT program. However, the potential roles and underlying mechanisms of miR-379-5p in regulating EMT of NPC cells remain unclear.

Methods: miR-379-5p expression levels in human NPC tissues and cell lines were detected via quantitative real-time PCR (qRT-PCR). Then, the correlations between miR-379-5p expression in NPC tissues and clinicopathologic features and patients' prognosis were analyzed. The effect of miR-379-5p on the expression of EMT markers in NPC cells was evaluated by Western blot and qRT-PCR. NPC cells' migration and invasion were evaluated in vitro by Transwell migration and invasion assays, respectively. The target of miR-379-5p was predicted with three publicly available databases and further validated with dualluciferase reporter assay, qRT-PCR, and Western blot.

Results: The expression of miR-379-5p was significantly decreased in NPC tissues, and its low expression was significantly associated with multiple unfavorable clinicopathological factors and poor prognosis of NPC patients. Meanwhile, miR-379-5p was downregulated in NPC cell lines, and its exotic expression inhibited EMT to reduce the migration and invasion of NPC cells. Furthermore, Y-box binding protein 1 (YBX1) was identified and validated as a direct target of miR-379-5p, and restoring YBX1 expression could reverse the inhibitive effect of miR-379-5p on NPC cell EMT, migration and invasion.

Conclusion: Taken together, our findings indicate that miR-379-5p inhibits the EMT of NPC cells to reduce their migration and invasion abilities by post-transcriptionally suppressing YBX1 expression, providing a novel potential treatment target for NPC patients.

Keywords: miR-379-5p, nasopharyngeal carcinoma, YBX1, EMT, migration, invasion

\section{Introduction}

Nasopharyngeal carcinoma (NPC), as an aggressive tumor originated from nasopharyngeal epithelial cells, is most prevalent in Southern China, accounting for $47.7 \%$ of newly diagnosed cases worldwide. ${ }^{1}$ During the past years, with the advancement of concurrent chemoradiotherapy and immunotherapy strategies, the clinical outcome of NPC patients has been obviously improved. ${ }^{2}$ However, tumor metastasis remains the leading cause of treatment failure for NPC patients. ${ }^{2}$ Recently, epithelial-mesenchymal transition (EMT) has been demonstrated to be a critical factor for modulating NPC metastasis. ${ }^{3-5}$ Through EMT program, 
epithelial tumor cells transform to a mesenchymal phenotype which is characterized by changes in cell morphology, a loss of epithelial cell polarity, disruption of cell-cell adhesion, and the acquisition of increased migratory and invasive capability, there mediating distant metastasis of epithelial malignant tumors. ${ }^{5}$ Therefore, exploring the underlying mechanisms of NPC cells' EMT has great significance for further understanding of the metastatic process in NPC.

The initiation and maintenance of EMT status are a multi-step complex processes cooperating by multiple factors and genes. ${ }^{6,7}$ Increasing and accumulating studies have demonstrated that microRNAs (miRNAs, miRs) play important roles in regulating NPC cells' EMT. ${ }^{8-10}$ As a large class of small endogenous non-coding RNAs (20-24nt in length), miRNAs function as the posttranscriptional regulators for EMT-related genes expression by completely or incompletely binding to the 3 '-untranslated regions (3'-UTRs) of their messenger RNA (mRNA) to mediate their degradation or suppress translation, thereby involving in the EMT process. ${ }^{8,10}$ MiR-379-5p, as a number of miR-379 family, is located on the human chromosome region 14q32.31. A growing body of evidence has indicated that abnormal expression of miR-379-5p participated in the development and progression of various human cancers. ${ }^{1-14}$ To date, miR-379-5p has been observed to be down-regulated in multiple types of solid tumors, which serves as a tumor suppressor through directly suppressing diverse cancerrelated genes expression to inhibit the EMT, proliferation, migration and invasion of tumor cells. ${ }^{11-14}$ For example, Chen et al and $\mathrm{Xu}$ et al showed that miR-379$5 p$ could inhibit the EMT and metastasis of hepatocellular carcinoma and gastric cancer by targeting FAK/AKT signaling axis; ${ }^{11,13}$ miR-379-5p suppressed the growth and metastasis of bladder cancer by directly targeting MDM2. ${ }^{12}$ However, the expression profile of miR-379$5 \mathrm{p}$ in NPC, and its roles and underlying mechanisms in regulating EMT of NPC cells have not yet been identified.

Y-box binding protein-1 (YBX1) is a multifunctional protein encoded by the YBX1 gene with an evolutionary conserved cold shock domain, ${ }^{15}$ which regulates transcription, translation, DNA damage repair, and other biological processes that occur in both the nucleus and cytoplasm. ${ }^{16}$ Nowadays, emerging and increasing evidence has shown that high levels of YBX1 protein were closely related to tumor metastasis and poor prognosis in various human cancers, which plays an oncogenic role in tumor development and progression by involving in the proliferation, growth, survival, and drug resistance of tumor cells, and chromatin destabilization by cancer. ${ }^{17-19}$ Moreover, in NPC, high YBX1 expression was demonstrated to be closely associated with poor prognosis of NPC patients, which promoted the migration and invasion of NPC cells by regulating EMT program. ${ }^{20,21}$ However, the molecular mechanisms of YBX1 promoting EMT in NPC cells remain largely unknown.

Therefore, in the present study, we explored the role and potential mechanism of miR-379-5p in NPC via clinical sample analysis and in vitro experiments. The results found that miR-379-5p was down-regulated in NPC tissues, and its low expression was significantly correlated with multiple unfavorable clinicopathological factors and poor prognosis of NPC patients. Functional assays showed that overexpression of miR-379-5p inhibited the EMT to reduce migration and invasion of NPC cells in vitro while silencing miR-379-5p exhibited an opposite function. Further mechanism studies indicated that YBX1 was a direct target of miR-379-5p, and restoration of YBX1 expression could reverse the inhibitive effect of miR-379$5 p$ overexpression on NPC cells' EMT, migration and invasion. These findings suggest that miR-379-5p functions as a tumor suppressor in NPC, providing a novel potential treatment target for NPC.

\section{Materials and Methods}

\section{Human Tissue Samples}

All tissue samples (including 95 NPC tissues and 50 normal nasopharyngeal epithelium tissues) were collected from the patients who were inpatient or outpatient treatment in Maternal and Child Health Care Hospital of Hubei Province and Zhongnan Hospital of Wuhan University from January 2011 to December 2012. Inclusion criteria: 1) histopathologically confirmed as NPC; 2) did not receive any anti-tumor treatment; 3 ) with complete clinicopathological data and follow-up information. Exclusion criteria: accompanied by other malignant tumors, cardiovascular and cerebrovascular diseases, infectious diseases, or history of alcohol abuse and mental illness. All tissue specimens were immediately collected after resection and stored at $-80{ }^{\circ} \mathrm{C}$ or embedded in paraffin. NPCs were graded using the World Health Organization grading system and staged according to the seventh edition of the American Joint Committee on Cancer (AJCC) tumor- 
node- metastasis (TNM) staging system. This study was approved by the Medical Ethics Committees of Maternal and Child Health Care Hospital of Hubei Province and Zhongnan Hospital of Wuhan University, and conducted in accordance with the ethical guidelines of the Declaration of Helsinki. Written informed consent was obtained from all included patients.

\section{Cell Culture and Transfection}

The human NPC cell lines (5-8F, C666-1, CHNE1, SUNE1 and CNE2) and human immortalized nasopharyngeal epithelial cell line (NP69) were purchased from the Chinese Academy of Sciences in Shanghai (Shanghai, China). NPC cells were cultured under standard conditions in RPMI-1640 Medium (Gibco, USA) supplemented with 10\% fetal bovine serum (FBS, Gibco), while NP69 was cultured in KSFM medium (Gibco) supplemented with $10 \%$ FBS. All cell lines were maintained in a humidified atmosphere at $37^{\circ} \mathrm{C}$ with $5 \% \mathrm{CO}_{2}$. The mimics and inhibitor of miR-379-5p were synthesized by Riobio, and Vigene (Shandong, China) offered plasmid of YBX1 (p-YBX1) and si-YBX1. The oligonucleotide transfection was conducted with Lipofectamine 2000 reagent (Invitrogen, Carlsbad, CA, USA), according to the manufacturer's instructions.

\section{RNA Isolation and Quantitative RT-PCR (qRT-PCR)}

Total RNA was extracted using Trizol reagent (Invitrogen, CA, USA), and reverse-transcription was conducted with MicroRNA Reverse Transcription kit (RioBio, Guangzhou, China) or mRNA Reverse Transcription kit (TaKaRa, Japan). PCR assays were carried out using a 7500 Real-Time PCR System (Applied Biosystems, CA, USA), and samples were normalized to U6 or GAPDH. The relative expression was calculated using the $2^{-\Delta \Delta \mathrm{CT}}$ method. The primers used in the present study were as follows: miR-379-5p, 5'-GCG CTGGTAGACTATGGAA-3' and 5'-GTGCAGGGTCCGA GGT-3'; U6, 5'-CTCGCTTCGGCAGCACA-3' and 5'-AAC GCTTCACGAATTTGCGT-3'; YBX1, 5'-TAGACGCTATC CACGTCGTAG-3' and 5'-ATCCCTCGTTCTTTTCCCCA C-3'; E-cadherin, 5'-ATTTTTCCCTCGACACCCGAT-3' and 5'-TCCCAGGCGTAGACCAAGA-3'; Vimentin, 5'GACGCCATCAACACCGAGTT-3' and 5'-CTTTGTCGTT GGTTAGCTGGT-3'; GAPDH, 5'-CTGGGCTACACTGAG CACC-3' and 5'-AAGTGGTCGTTGAGGGCAATG-3'.

\section{Western Blot}

Total protein was extracted from the cells, and electrophoresed through a $10 \%$ denaturing polyacrylamide gel. Protein was then transferred to PVDF membranes (Merck Millipore, MA, USA), and blocked in 5\% bovine serum albumin for $1 \mathrm{~h}$, followed by incubated with the primary antibodies at $4{ }^{\circ} \mathrm{C}$ overnight and secondary antibodies for $1 \mathrm{~h}$ at room temperature. The Chemiluminescence Western Detection System (BioRad, CA, USA) was used to detect the relative protein band intensity. The following primary antibodies were used: antiE-cadherin (Abcam, USA), anti-Vimentin (Abcam, USA), anti-YBX1 (Abcam, USA), and anti-GAPDH (Abcam, USA).

\section{Transwell Migration and Invasion Assay}

The migration and invasion assays were performed in a 24well plate with $8-\mathrm{mm}$ pore size and $6.5-\mathrm{mm}$ diameter Transwell chambers (Corning, NY, USA). For cell migration assay, transfected C666-1 and CNE-1 in FBS-free medium were plated in the upper chamber and medium containing FBS was added to the lower wells. Cells that had migrated to the lower chamber were stained with $0.1 \%$ crystal violet after $24 \mathrm{~h}$ of incubation at $37^{\circ} \mathrm{C}$. Then, cells of the lower chamber were fixed and stained by using $4 \%$ paraformaldehyde and crystal violet, respectively, and the stained cells were photographed and counted using a light microscope. The invasion assay was performed according to the procedure of the cell migration assay, except that the invasion chambers were pre-coated with Matrigel (BD Biosciences, USA).

\section{Dual-Luciferase Reporter Assay}

For the luciferase reporter assay, a fragment of YBX1 gene 3'UTR (WT-3'UTR) containing a miR-379-5p targeting site was amplified by PCR and cloned into psiCHECK $^{\mathrm{TM}}$ vector (Promega, WI, USA). Then, C666-1 and CNE-1 were cotransfected with wild type or mutant 3'UTR reporter and miR-379-5p mimics or miR-379-5p inhibitor, respectively, and the Renilla luciferase activities were detected by the Dual Luciferase Assay (Promega, WI, USA).

\section{Statistical Analysis}

All statistical analyses were performed using SPSS 22.0.0 (SPSS Statistics, IL, USA) and GraphPad Prism 6.0 (GraphPad Software Inc., CA, USA) for Windows. Continuous data was expressed as the means \pm standard deviations (SD), and differences between groups were compared using Student's $t$-test or ANOVA, as appropriate. The associations between miR-379-5p expression and 
clinicopathological parameters were tested using the Chisquare $\left(\chi^{2}\right)$ or Fisher's exact test. The correlation between the miR-379-5p and YBX1 mRNA levels in NPC tissues was determined using the Spearman correlation test. Kaplan-Meier method was used for survival analysis and drawing the survival curves, and difference among patients' subgroups was calculated by Log-rank test. Univariate and multivariate Cox-regression analyses were applied to identify the independent factors affecting the prognosis of NPC patients. All experiments for cell cultures were performed independently at least three times. In all cases, $P$ values $<0.05$ were considered statistically significant.

\section{Results}

\section{Downregulation of miR-379-5p Correlates with Malignant Progression and Poor Prognosis of NPC}

To explore the role of miR-379-5p in the progression of human NPC, we first detected the expression level of miR-379-5p in human NPC and normal nasopharyngeal

\section{A}
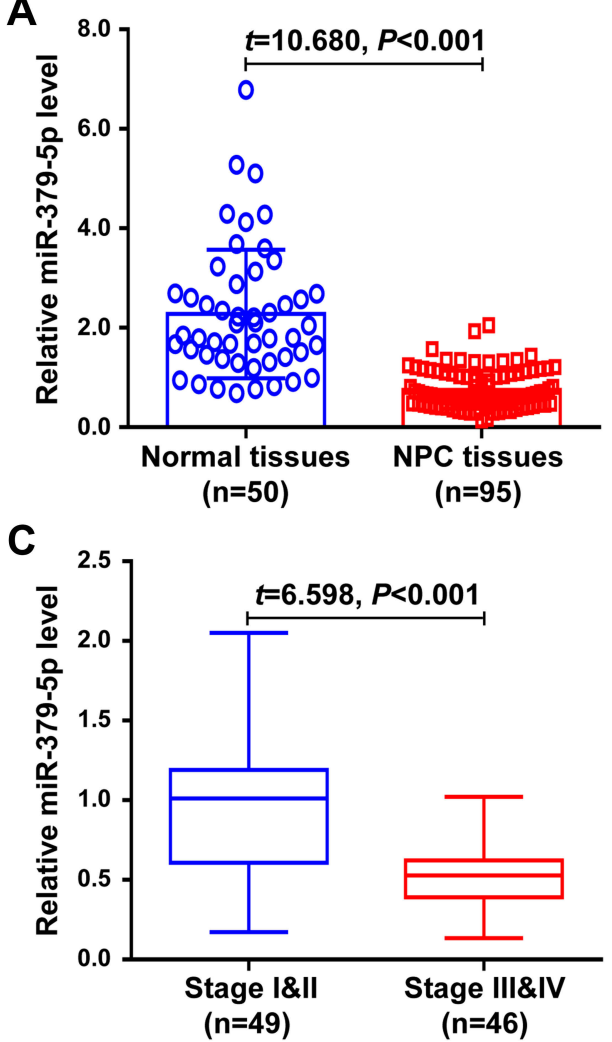

epithelium tissues. The results showed that miR-379-5p was significantly downregulated in NPC tissues compared with normal nasopharyngeal epithelium tissues $(t=10.680, \quad P<0.001, \quad$ Figure $1 \mathrm{~A})$, and miR-379-5p expression was markedly decreased in patients with poor tumor grade $(t=7.778, P<0.001$, Figure 1B) and advanced TNM stage $(t=6.598, P<0.001$, Figure $1 \mathrm{C})$. Furthermore, we analyzed the relationships between miR-379-5p expression in NPC tissues and the clinicopathological features of patients. The results found that the low expression of miR-379-5p was significantly associated with tumor grade, $\mathrm{T}$ stage, $\mathrm{N}$ stage and TNM stage $(P<0.05$, respectively), but not with gender, age and Epstein-Barr virus (EBV) status $(P>0.05$, respectively, Table 1). Further prognostic analysis revealed that a low level of miR-379-5p expression was significantly associated with poor overall survival (OS) of NPC patients $\left(\chi^{2}=6.927, P=0.008\right.$, Figure 1D). Univariate and multivariate Cox analyses demonstrated that low miR-379-5p expression was an independent prognostic factor for affecting patient's $\mathrm{OS}(\mathrm{HR}=0.689$,

B

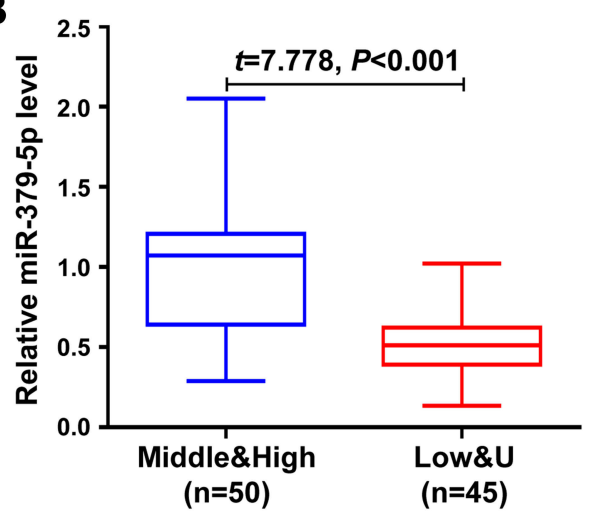

D

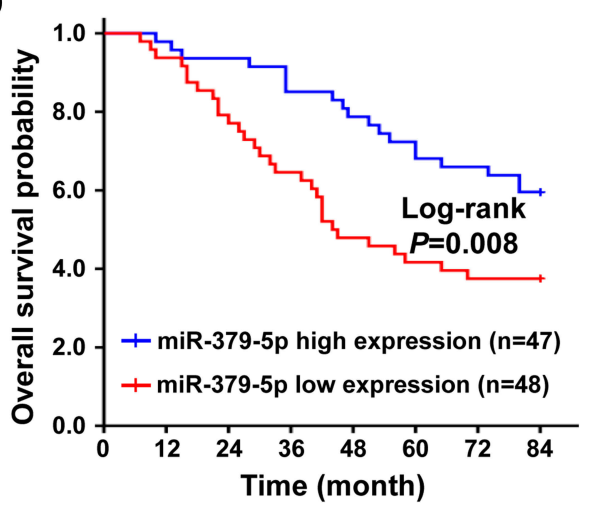

Figure I Downregulation of miR-379-5p correlates with malignant progression and poor prognosis of NPC. (A) qRT-PCR analysis of the expression levels of miR-379-5p in NPC and normal nasopharyngeal epithelium tissues. (B) Relative expression of miR-379-5p in different grades of NPC tissues. (C) Relative expression of miR-379-5p in different stages of NPC tissues. (D) The relationship between miR-379-5p expression in NPC tissues and overall survival of patients.

Abbreviations: NPC, nasopharyngeal carcinoma; qRT-PCR, quantitative real-time PCR.m 
Table I Relationships Between miR-379-5p Expression in NPC Tissues and Patients' Clinicopathological Features $(n=95)$

\begin{tabular}{|c|c|c|c|c|c|}
\hline \multirow[t]{2}{*}{ Parameter } & \multirow[t]{2}{*}{ Case } & \multicolumn{2}{|c|}{$\begin{array}{l}\text { miR-379-5p } \\
\text { Expression }\end{array}$} & \multirow[t]{2}{*}{$\begin{array}{l}\chi^{2} \\
\text { value }\end{array}$} & \multirow[t]{2}{*}{$P$ value } \\
\hline & & $\begin{array}{l}\text { Low } \\
(n=48)\end{array}$ & $\begin{array}{l}\text { High } \\
(n=47)\end{array}$ & & \\
\hline $\begin{array}{l}\text { Gender } \\
\text { Male } \\
\text { Female }\end{array}$ & $\begin{array}{l}61 \\
34\end{array}$ & $\begin{array}{l}34 \\
14\end{array}$ & $\begin{array}{l}27 \\
20\end{array}$ & 1.852 & 0.174 \\
\hline $\begin{array}{l}\text { Age (years) } \\
\quad \leq 60 \\
>60\end{array}$ & $\begin{array}{l}60 \\
35\end{array}$ & $\begin{array}{l}32 \\
16\end{array}$ & $\begin{array}{l}28 \\
19\end{array}$ & 0.513 & 0.474 \\
\hline $\begin{array}{l}\text { Tumor grade } \\
\text { Middle\&High } \\
\text { Low\&U }\end{array}$ & $\begin{array}{l}50 \\
45\end{array}$ & $\begin{array}{l}19 \\
29\end{array}$ & $\begin{array}{l}31 \\
16\end{array}$ & 6.626 & $0.010 *$ \\
\hline $\begin{array}{l}\text { T stage } \\
\text { TI\&T2 } \\
\text { T3\&T4 }\end{array}$ & $\begin{array}{l}36 \\
59\end{array}$ & $\begin{array}{l}13 \\
35\end{array}$ & $\begin{array}{l}23 \\
24\end{array}$ & 4.819 & $0.028 *$ \\
\hline $\begin{array}{l}\text { N stage } \\
\text { N0\&NI } \\
\text { N2\&N3 }\end{array}$ & $\begin{array}{l}57 \\
38\end{array}$ & $\begin{array}{l}24 \\
24\end{array}$ & $\begin{array}{l}33 \\
14\end{array}$ & 4.043 & $0.044 *$ \\
\hline $\begin{array}{l}\text { TNM stage } \\
\text { I\&II } \\
\text { III\&IV }\end{array}$ & $\begin{array}{l}49 \\
46\end{array}$ & $\begin{array}{l}18 \\
30\end{array}$ & $\begin{array}{l}31 \\
16\end{array}$ & 7.700 & $0.006 *$ \\
\hline $\begin{array}{l}\text { EBV status } \\
\text { Negative } \\
\text { Positive }\end{array}$ & $\begin{array}{l}33 \\
62\end{array}$ & $\begin{array}{l}13 \\
35\end{array}$ & $\begin{array}{l}20 \\
27\end{array}$ & 2.507 & 0.113 \\
\hline
\end{tabular}

Note: $* P<0.05$.

Abbreviations: NPC, nasopharyngeal carcinoma; U, undifferentiated; TNM, tumor-node-metastasis; EBV, Epstein-Barr virus.

95\% CI $=0.343-0.923, P=0.028$, Table 2). These results indicate that miR-379-5p might play a tumorsuppressive role in NPC initiation and progression.
miR-379-5p Suppresses EMT Program to Reduce the Migratory and Invasive Abilities of NPC Cells

To further investigate the role of miR-379-5p in the development and progression of NPC, we analyzed the effect of ectopic expression of miR-379-5p on malignant behaviors of NPC cells. First, the levels of miR-379-5p expression in five human NPC cells and human immortalized nasopharyngeal epithelial cells were detected by qRT-PCR. The results showed that the miR-379-5p levels were markedly decreased in NPC cell lines compared with the human immortalized nasopharyngeal epithelial cells (Figure 2A), consistent with NPC tissues. Among them, CNE1 presented the highest miR-379-5p level while C666-1 presented the lowest level. Thus, C666-1 and CNE1 cells were respectively selected for transfection with miR-379$5 p$ mimics and miR-379-5p inhibitor for the gain- and loss-of-function assays. The results showed that miR$379-5 p$ mimics significantly increased the expression of miR-379-5p in C666-1 (Figure 2B), while miR-379-5p inhibitor had the opposite effect in CNE1 (Figure 2C).

Furthermore, we investigated whether miR-379-5p could regulate the EMT of NPC cells in vitro. Western blot showed that the epithelial marker-E-cadherin expression was increased while the mesenchymal markerVimentin expression was decreased in C666-1 cells after transfected with miR-379-5p mimics, by contrast, CNE1 cells transfected with miR-379-5p inhibitor exhibited opposite phenomenon (Figure 2D). Further qRT-PCR results confirmed that the levels of E-cadherin and Vimentin mRNAs were consistent with protein changes in C666-1 cells transfected with miR-379-5p mimics (Figure 2E), or CNE1 cells transfected with miR-379-5p

Table 2 Univariate and Multivariate Analyses of the Risk Factors for Overall Survival of NPC Patients

\begin{tabular}{|c|c|c|c|c|c|c|}
\hline \multirow[t]{2}{*}{ Parameter } & \multicolumn{3}{|c|}{ Univariate Analyses } & \multicolumn{3}{|c|}{ Multivariate Analyses } \\
\hline & HR & $95 \% \mathrm{Cl}$ & P value & HR & $95 \% \mathrm{Cl}$ & $P$ value \\
\hline Gender (Female vs Male) & 1.362 & $0.777-2.238$ & 0.281 & - & - & - \\
\hline Age ( $\leq 60$ vs $>60$ years $)$ & 1.292 & $0.710-2.348$ & 0.401 & - & - & - \\
\hline Tumor grade (Middle\&High vs Low\&U) & 1.862 & $1.042-3.333$ & $0.036^{*}$ & 1.503 & $0.822-2.755$ & 0.185 \\
\hline T stage (TI\&T2 vs T3\&T4) & 1.445 & $1.003-2.08 \mid$ & $0.048^{*}$ & 1.284 & $0.822-2.005$ & 0.272 \\
\hline $\mathrm{N}$ stage (N0\&NI vs N2\&N3) & 1.381 & $1.025-1.859$ & $0.034^{*}$ & 1.254 & $0.714-2.067$ & 0.183 \\
\hline TNM stage (I\&II vs III\&IV) & 6.320 & 4.062-9.834 & $<0.00 I^{*}$ & 11.894 & $6.338-22.319$ & $<0.00 I^{*}$ \\
\hline EBV status (Negative vs Positive) & 1.029 & $0.476-2.227$ & 0.942 & - & - & - \\
\hline miR-379-5p expression (Low vs High) & 0.471 & $0.264-0.838$ & $0.010^{*}$ & 0.689 & $0.343-0.923$ & $0.028^{*}$ \\
\hline
\end{tabular}

Notes: -Unavailable; $* P<0.05$.

Abbreviations: NPC, nasopharyngeal carcinoma; U, undifferentiated; TNM, tumor-node-metastasis; EBV, Epstein-Barr virus; HR, hazard ratio; Cl, confidence interval. 
A

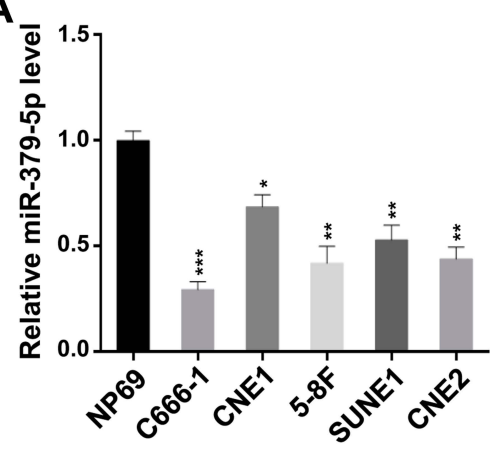

D

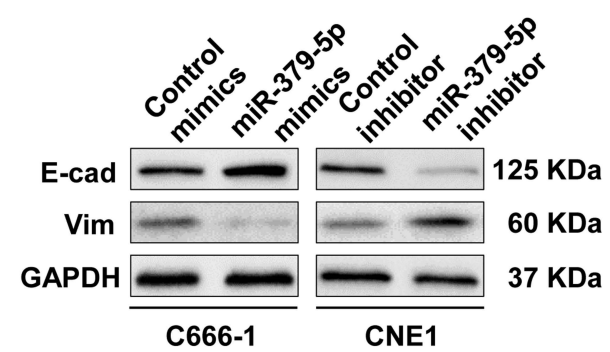

B

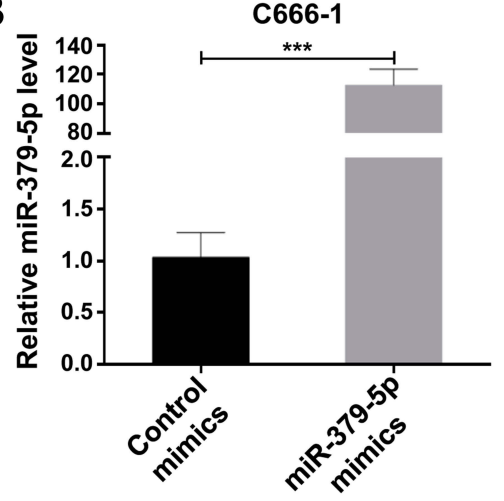

E

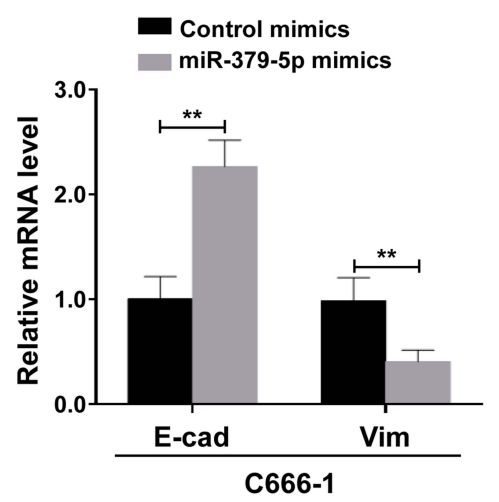

C

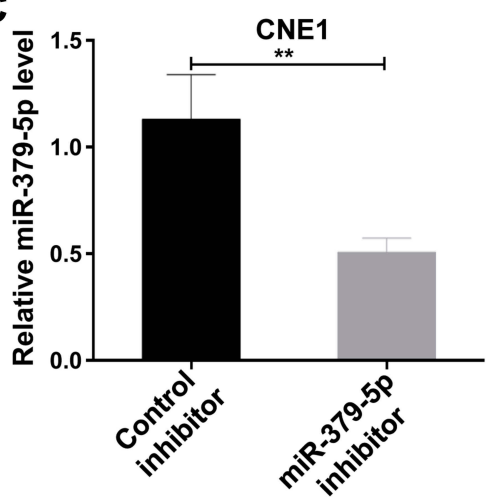

F

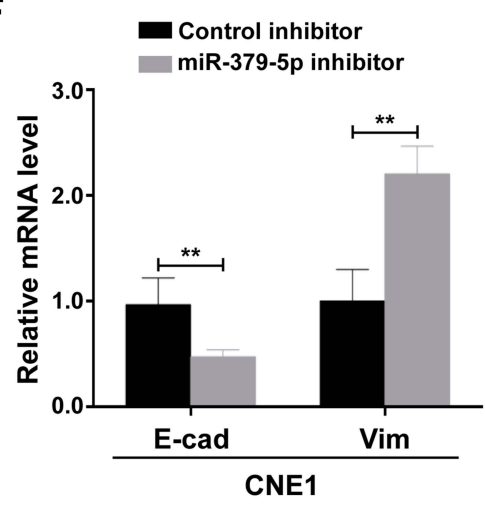

G

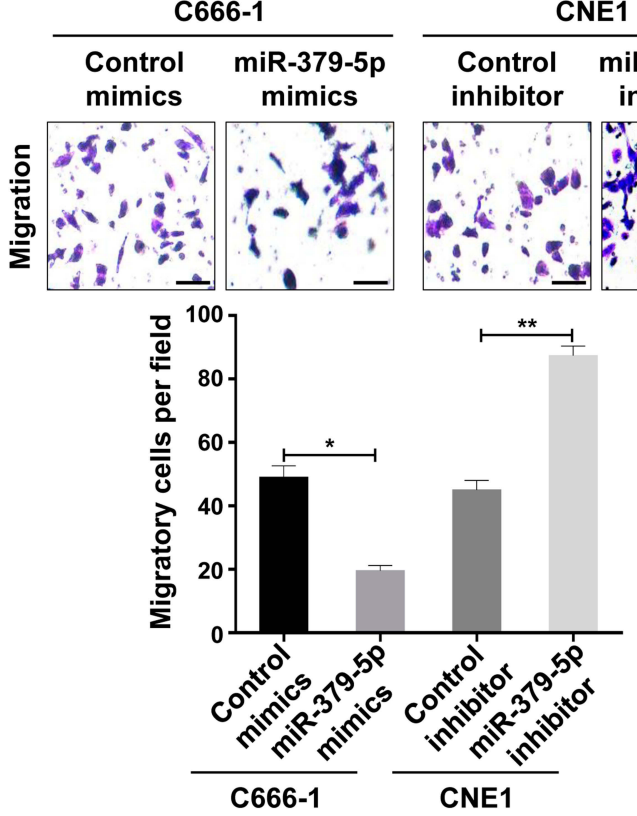

H
CNE1

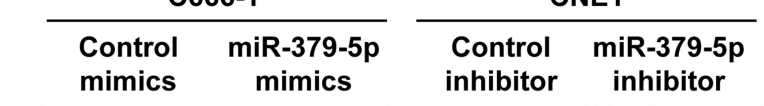

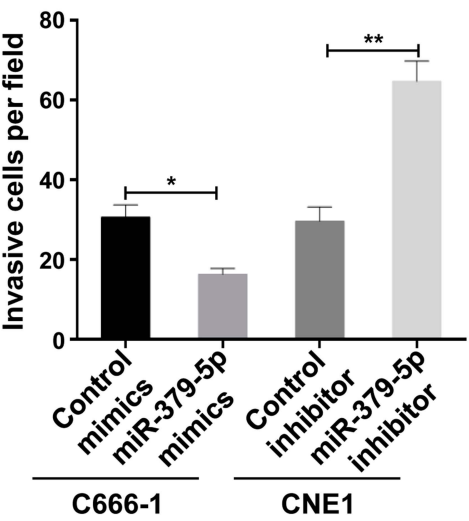

Figure 2 miR-379-5p suppresses EMT program to reduce the migratory and invasive abilities of NPC cells. (A) qRT-PCR analysis of the expression levels of miR-379-5p in various human NPC cell lines and human immortalized nasopharyngeal epithelial NP69 cells. (B, C) qRT-PCR analysis of the transfection efficiency in C666-I cells after transfection with miR-379-5p mimics or control mimics and in CNEI cells after transfection with miR-379-5p inhibitor or control inhibitor. (D) Western blot analysis of E-cadherin and Vimentin protein expression in C666-I and CNEI cells after, respectively, transfected with miR-379-5p mimics or inhibitor. (E, F) qRT-PCR analysis of E-cadherin and Vimentin mRNA expression in C666-I cells after transfected with miR-379-5p mimics. And in CNEI cells after transfected with miR-379-5p inhibitor. (G, H) The effects of miR-379-5p overexpression or knockdown on migration or invasion of C666-I and CNEI cells were analyzed using Transwell assays or Matrigel-coated Transwell assays, respectively. Scale bars, $100 \mu \mathrm{m}$.

Notes: $* P<0.05 ; * * P<0.01 ; * * * P<0.001$.

Abbreviations: NPC, nasopharyngeal carcinoma; EMT, epithelial-mesenchymal transition; E-cad, E-cadherin; Vim, Vimentin; qRT-PCR, quantitative real-time PCR. 
inhibitor (Figure 2F). These data suggest that miR-379-5p suppresses the EMT program of NPC cells.

Given the important role of EMT in tumor cell's migration and invasion, Transwell migration and invasion assays were further performed to determine the effect of miR-379$5 \mathrm{p}$ mimics or inhibitor on the migratory and invasive abilities of C666-1 or CNE1 cells, respectively. The results showed that overexpression of miR-379-5p suppressed the migration and invasion of C666-1 cells, while miR-379-5p knockdown increased the migratory and invasive abilities of CNE1 cells in vitro (Figure $2 \mathrm{G}$ and $\mathrm{H}$ ). Taken together, these findings indicate that miR-379-5p suppresses EMT to inhibit the migratory and invasive behaviors in NPC cells.

\section{YBXI Is Identified as a Direct Target of miR-379-5p in NPC Cells}

Because miRNAs exert their functions mainly by repressing the expression of target genes, ${ }^{8}$ thus, the potential targets of miR-379-5p were predicted by utilizing three publically online database (including miRDB, TarBase and TargetScan). Among these predicated common targets (Figure 3A), YBX1 was focused on because it has been demonstrated to be involved in EMT and progression of NPC. ${ }^{20,21}$ qRT-PCR was used to detect the levels of YBX1 expression in five human NPC cells and human immortalized nasopharyngeal epithelial cells. The results showed that the YBX1 was markedly upregulated in NPC cell lines compared with the human immortalized nasopharyngeal epithelial cells (Figure 3B). Then, TargetScan prediction revealed that the 3'-UTR of YBX1 mRNA contains a conserved binding site (17-23) for miR-379-5p (Figure 3C). Subsequently, the dual-luciferase reporter assays were performed to test whether miR-379-5p directly targets YBX1. The results showed that the overexpression of miR-379-5p markedly reduced the luciferase activity associated with the wild-type (WT) 3'-UTR of YBX1 reporter plasmid compared with control cells (Figure 3D), whereas miR-379-5p knockdown increased this activity (Figure 3E). In contrast, miR-379-5p did not affect the luciferase activity of the mutant-type (MT) 3'-UTR of YBX1 reporter plasmid (Figure 3D and E). These data suggest that miR-379-5p can directly bind to the 3 '-UTR of YBX1 mRNA.

To further confirm the above findings, Western blot and qRT-PCR were employed to evaluate the regulatory effects of miR-379-5p on endogenous YBX1 protein and mRNA expression in the C666-1 and CNE1 cells. The results showed that the expression of YBX1 protein was significantly decreased in C666-1 cells transfected with miR-379-5p mimics compared with control cells, whereas YBX1 protein expression was significantly increased in CNE1 cells transfected with miR-379-5p inhibitor (Figure 3F). Meanwhile, qRT-PCR demonstrated that the expression of YBX1 mRNA was also significantly affected by the overexpression or inhibition of miR-379-5p in both C6661 and CNE1 cells (Figure 3G and H), which was consistent with the changes of YBX1 protein. Moreover, Spearman correlation analysis indicated an inverse correlation between miR-379-5p and YBX1 mRNA expression in the human NPC tissues ( $r=-0.4689, P<0.001$; Figure $3 \mathrm{I})$. Collectively, these findings indicate that YBX1 is a direct target of miR379-5p and that miR-379- 5p post-transcriptionally suppresses the expression of YBX1 in NPC cells by directly binding to the $3^{\prime}$-UTR of its mRNA to degrade it.

\section{Repression of YBXI is Essential for the miR-379-5p-Mediated Inhibition of EMT, Migration and Invasion in NPC Cells}

Then, we assessed whether YBX1 was required for the regulatory effect of miR-379-5p on NPC cells' EMT, migration and invasion. To validate this hypothesis, YBX1 plasmid along with miR-379-5p mimics were firstly co-transfected into C666-1 cells. As expected, Western blot showed that the ectopic overexpression of YBX1 reversed miR-379-5p overexpression-mediated YBX1 protein repression in C666-1 cells (Figure 4A). This change was accompanied by increased Vimentin expression and decreased E-cadherin expression (Figure 4A). Further qRT-PCR results also confirmed the above findings (Figure 4B). Furthermore, as observed in Transwell migration and invasion assays, YBX1 overexpression also abolished miR-379-5p overexpression-mediated inhibitive effects of migration and invasion in C666-1 cells (Figure 4C and D). Then, we further co-transfected si-YBX1 and miR-379-5p inhibitor into CNE1 cells. The results showed that repression of the expression of YBX1 could rescue the effect of miR-379-5p inhibitor-mediated promotion of NPC cells' EMT, migration and invasion (Figure 5A-D). Together, these data suggest that the repression of YBX1 is essential for the miR-379-5p-mediated inhibition of EMT, migration and invasion in NPC cells.

\section{Discussion}

Tumor metastasis is the major cause of treatment failure and death for patients with NPC, ${ }^{2}$ which is a complex and 
A

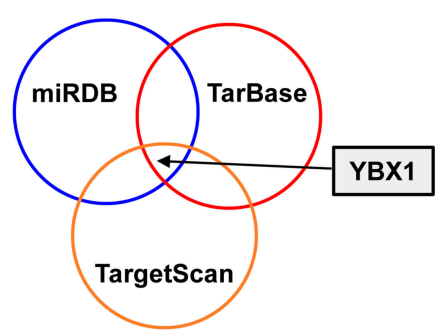

D

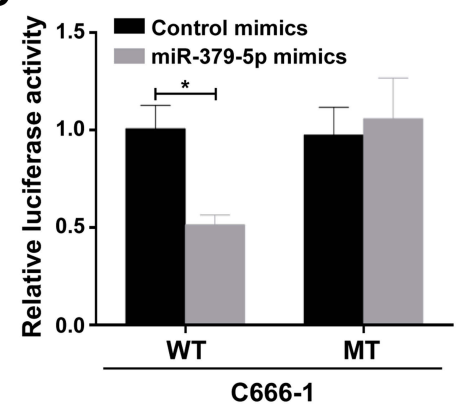

G

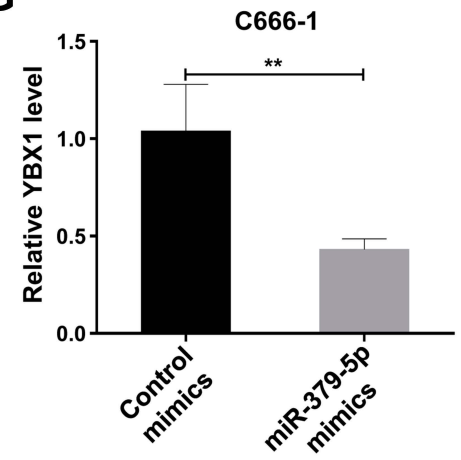

B

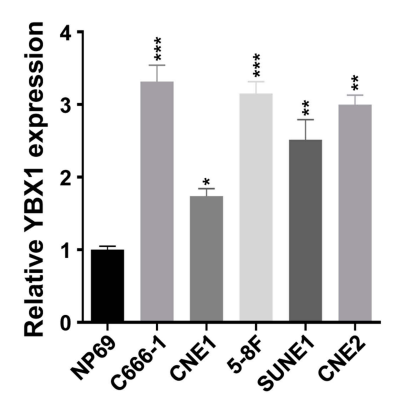

C

YBX1 mRNA

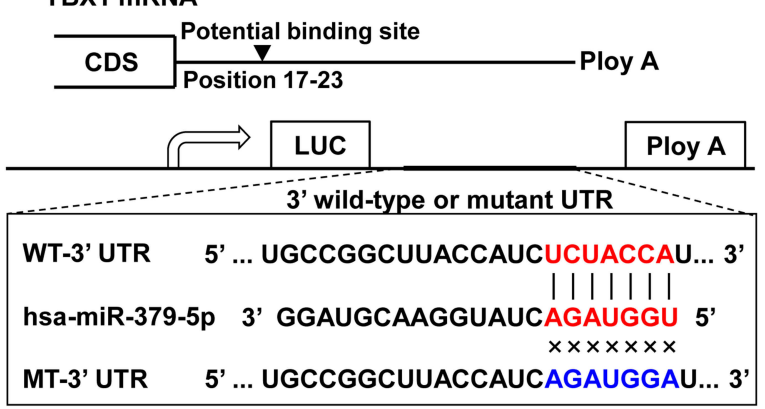

F
E

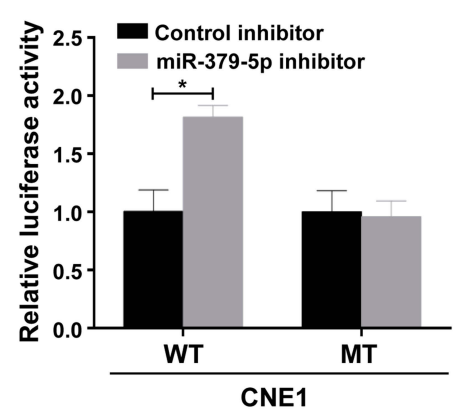

H

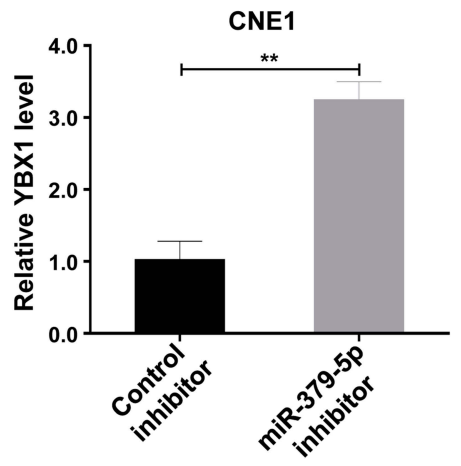

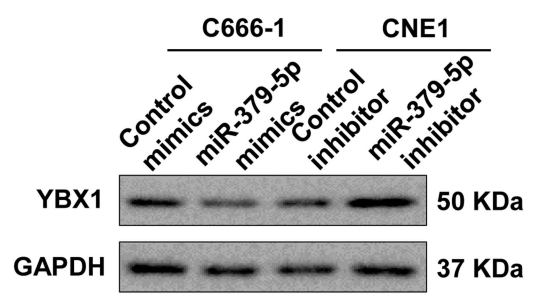

I

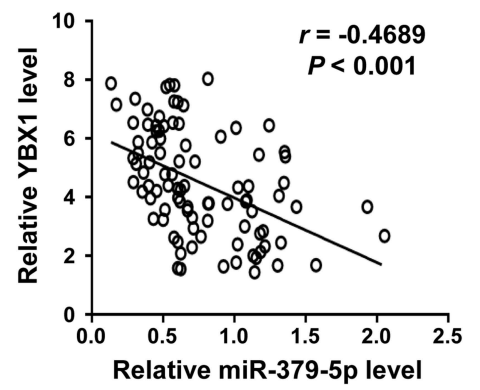

Figure 3 YBXI is identified as a direct target of miR-379-5p in NPC cells. (A) YBXI was predicted as a potential target of miR-379-5p by utilizing three publically online database, including miRDB, TarBase and TargetScan. (B) qRT-PCR analysis of the expression levels of YBXI in various human NPC cell lines and human immortalized nasopharyngeal epithelial NP69 cells. (C) Schematic of miR-379-5p putative binding sites in WT 3' UTR of YBXI and MT 3'-UTR of YBXI. (D) Relative luciferase activity of YBXI 3'-UTR in C666-I cells co-transfected with the indicated reporters and miR-379-5p mimics oligonucleotides. (E) Relative luciferase activity of YBXI 3'-UTR in CNEI cells co-transfected with the indicated reporters and miR-379-5p inhibitor oligonucleotides. (F) Western blot analysis of the protein levels of YBXI in C666-I cells transfected with miR-379-5p mimics and CNEI cells transfected with miR-379-5p inhibitor. (G, H) qRT-PCR analysis of the mRNA levels of YBXI in C666-I cells transfected with miR-379-5p mimics and CNEI cells transfected with miR-379-5p inhibitor. (I) Spearman's analysis of the correlation between miR-379-5p and YBXI mRNA expression in human NPC tissues.

Notes: $* P<0.05 ; * * P<0.01 ; * * * P<0.001$.

Abbreviations: NPC, nasopharyngeal carcinoma; YBXI, Y-box binding protein I; mRNA, messenger RNA; WT, wild-type; MT, mutant; 3'-UTR, 3'-untranslated region; qRTPCR, quantitative real-time PCR.

multistep process regulated by various molecules, ${ }^{5}$ including miRNAs. ${ }^{22}$ An increasing amount of evidence has demonstrated that miRNAs play crucial roles in multiple pathological processes associated with NPC onset and progression, such as cell proliferation, migration, invasion and metastasis. ${ }^{23,24}$ Furthermore, miRNAs have been proposed as novel prognostic markers and potential therapeutic targets for NPC. ${ }^{22,23}$ Therefore, further exploring the roles and molecular mechanisms of
miRNAs during NPC progression is particularly necessary. In the present study, we reported that miR-379-5p was down-regulated in NPC, and its low expression was significantly associated with multiple unfavorable clinicopathological factors and poor prognosis of NPC patients. Further in vitro experiments showed that miR379-5p could inhibit the EMT of NPC cells by posttranscriptionally suppressing YBX1 expression to reduce cell migrative and invasive capacities. These results 
A

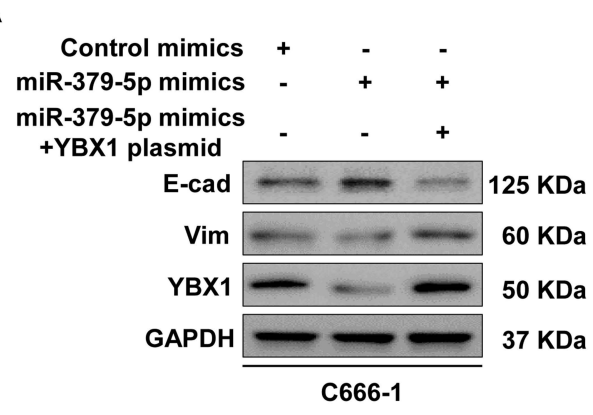

C

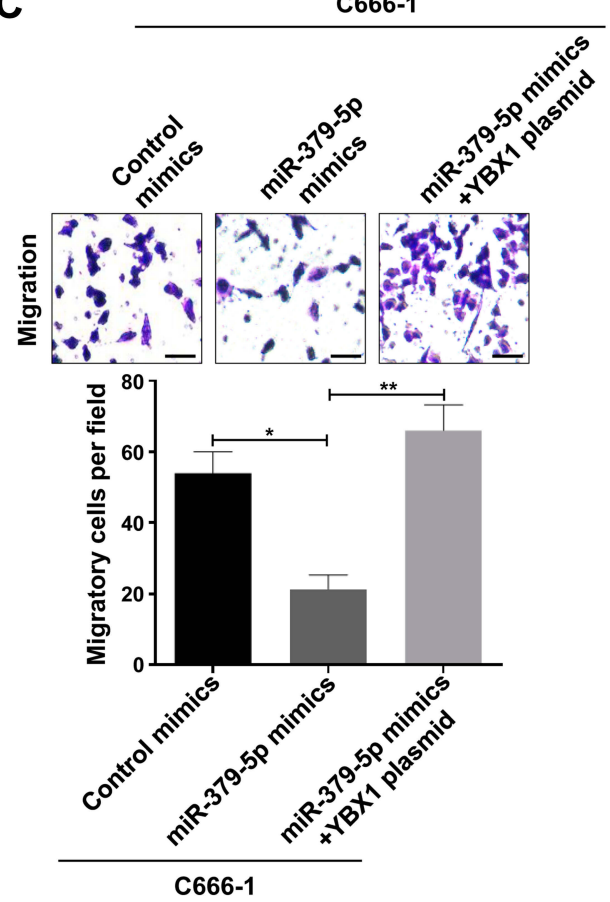

B

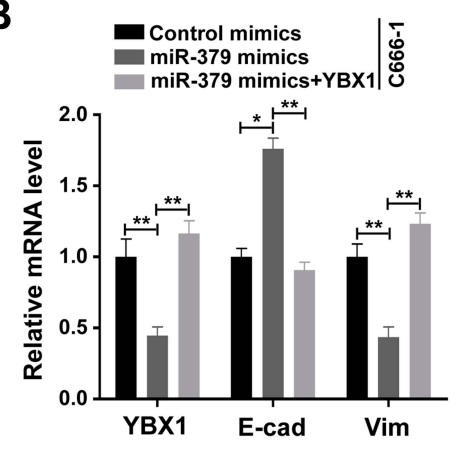

D

C666-1

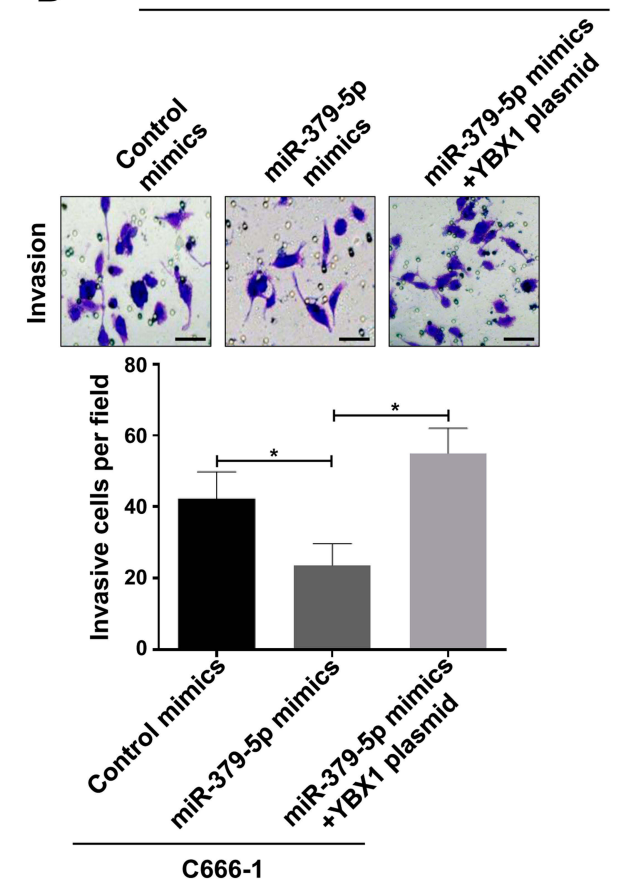

Figure 4 YBXI overexpression can rescue miR-379-5p mimics-mediated inhibition of EMT, migration and invasion in C666-I cells. (A) Western blot analysis of indicated protein expression in C666-I cells co-transfected with miR-379-5p mimics and YBXI plasmid. (B) qRT-PCR analysis of indicated mRNAs expression in C666-I cells cotransfected with miR-379-5p mimics and YBXI plasmid. (C-D) The effects of co-transfection of miR-379-5p mimics and YBXI plasmid on migration or invasion of C666-I cells were analyzed using Transwell assays or Matrigel-coated Transwell assays, respectively. Scale bars, $100 \mu \mathrm{m}$.

Notes: $* P<0.05 ; * * P<0.01$.

Abbreviations: NPC, nasopharyngeal carcinoma; YBXI, Y-box binding protein I; EMT, epithelial-mesenchymal transition; E-cad, E-cadherin; Vim, Vimentin; qRT-PCR, quantitative real-time PCR.

suggest that miR-379-5p functions as a tumor suppressor in NPC and may be investigated as a novel potential treatment target for clinical application. To the best of our knowledge, this study provides the first investigation to the underlying mechanism of miR-379-5p in regulating EMT-mediated progression of NPC.

Previously, miR-379-5p has been shown to be downregulated in many types of cancers and be associated with tumors malignant progression. For instance, Chen et al found that miR-379-5p was significantly downregulated in hepatocellular carcinoma (HCC), and its low expression correlated with advanced TNM stage and metastasis.
Further mechanistic studies demonstrated that miR-379$5 \mathrm{p}$ inhibited the migration, invasion, EMT and metastasis of HCC cells by targeting FAK/AKT signaling. ${ }^{11} \mathrm{Wu}$ et al showed that miR-379-5p was under-expressed in bladder cancer tissues compared with that in adjacent normal tissues. $^{12} \mathrm{Ji}$ et al found miR-379-5p expression was decreased in breast cancer tissues compared with that in normal breast tissues by analyzing the TCGA database. ${ }^{25}$ Herein, we showed that miR-379-5p was significantly down-regulated in both NPC tissues and cell lines, and low miR-379-5p expression was correlated with multiple unfavorable clinicopathological parameters, including 
A

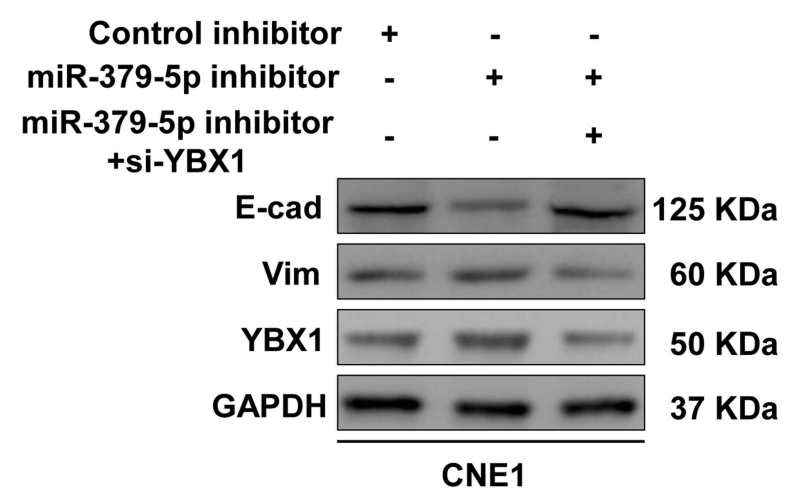

C

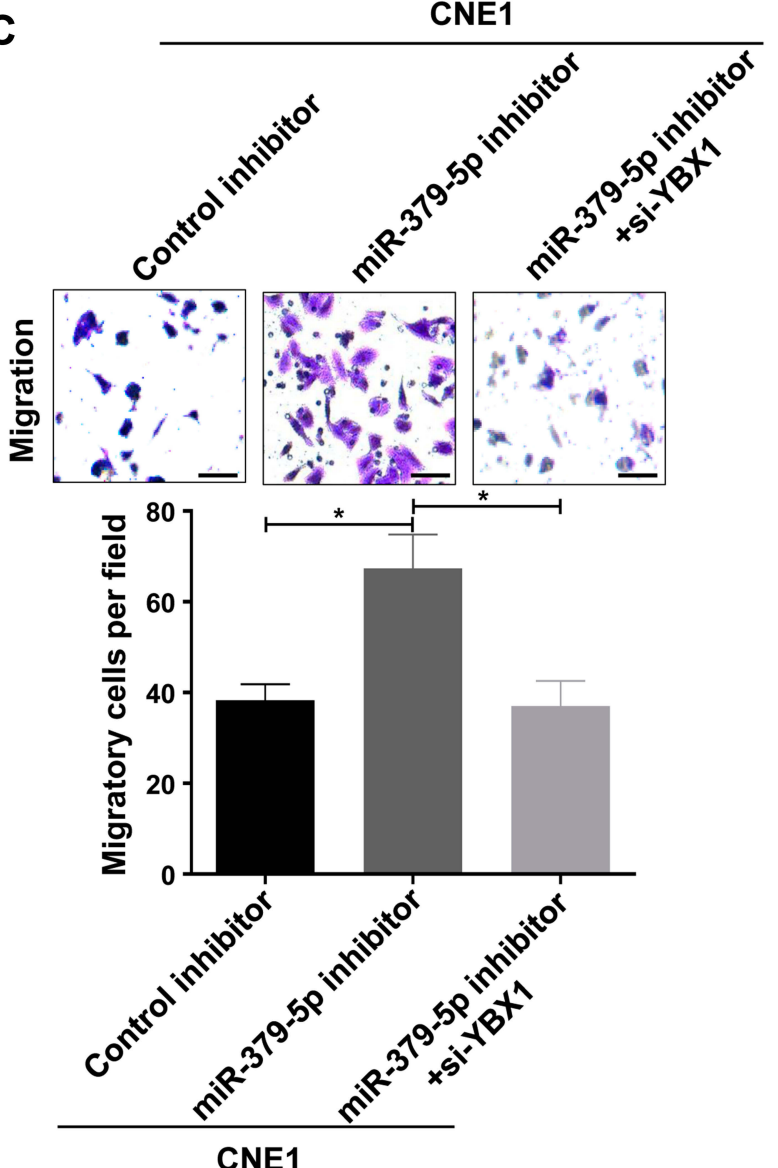

B
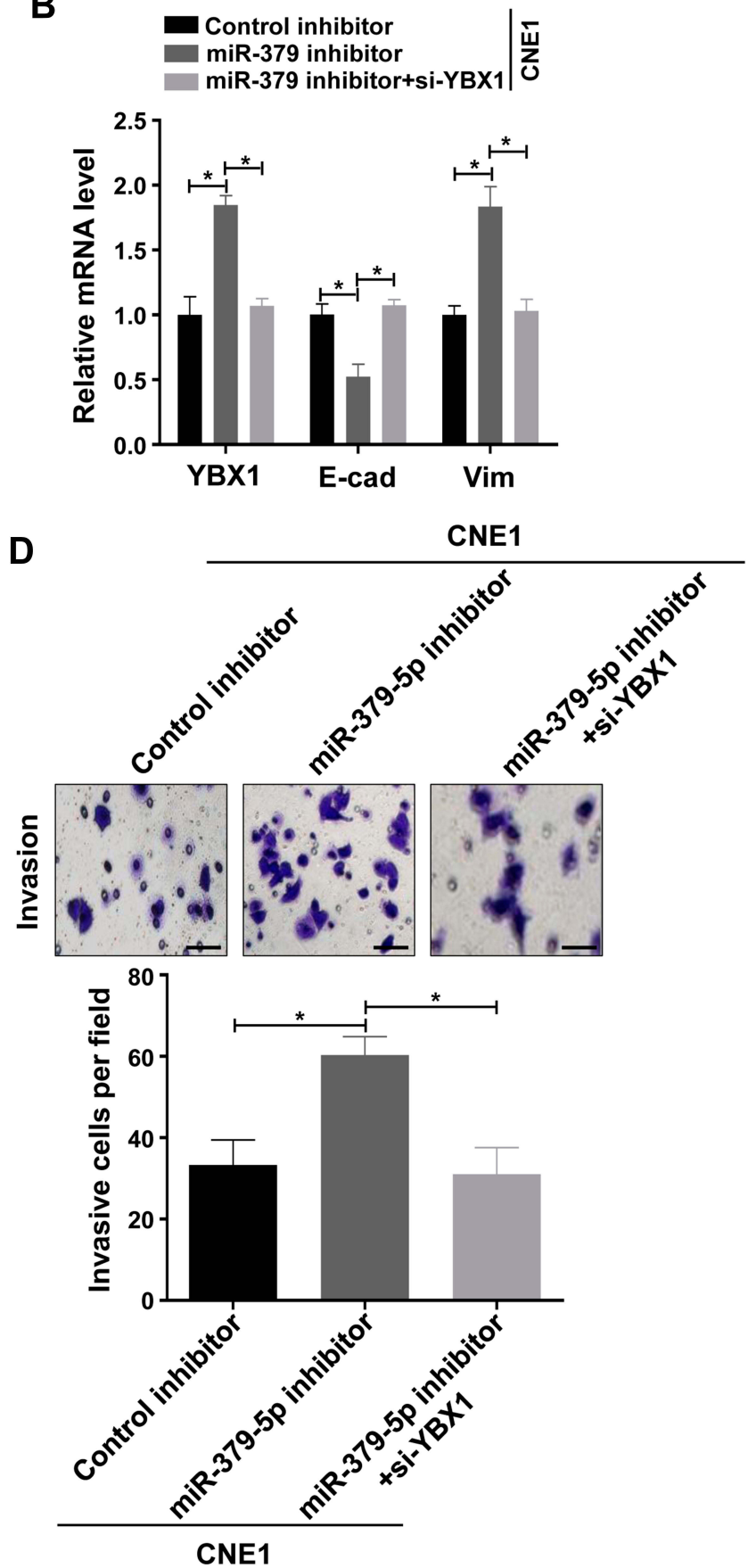

Figure 5 YBXI repression can rescue miR-379-5p inhibitor-mediated promotion of EMT, migration and invasion in CNEI cells. (A) Western blot analysis of indicated protein expression in CNEI cells co-transfected with miR-379-5p inhibitor and si-YBXI. (B) qRT-PCR analysis of indicated mRNAs expression in CNEI cells co-transfected with miR-379-5p inhibitor and si-YBXI. (C-D) The effects of co-transfection of miR-379-5p inhibitor and si-YBXI on migration or invasion of CNEI cells were analyzed using Transwell assays or Matrigel-coated Transwell assays, respectively. Scale bars, $100 \mu \mathrm{m}$.

Note: $* p<0.05$.

Abbreviations: NPC, nasopharyngeal carcinoma; YBXI, Y-box binding protein I; EMT, epithelialmesenchymal transition; E-cad, E-cadherin; Vim, Vimentin; qRT-PCR, quantitative real-time PCR.

tumor grade, T stage, $\mathrm{N}$ stage and TNM stage. Further survival analyses indicated that low miR-379-5p expression was an independent factor for affecting the poor OS of NPC patients. Together, these results suggest that miR-
$379-5 p$ might play a tumor-suppressive role in the development and progression of human cancers.

EMT, characterized by loss of E-cadherin, acquisition of Vimentin, and enhancement of migrative and invasive 
abilities, has been proposed as the key step for the metastatic progression of cancer. ${ }^{6,7}$ Recently, the roles of miRNAs in NPC cells' EMT have gained more attraction. For example, miR-9-3p suppressed the EMT process via downregulating FN1, ITGB1, ITGAV expression to reduce the proliferation and metastases of NPC; ${ }^{26}$ miR-BART8 $-3 p$ involved in EMT of NPC cells through directly targeting RNF38 to activate $\mathrm{NF}-\kappa \mathrm{B}$ and Erk1/2 signaling axis, thereby promoting NPC progression and metastasis; ${ }^{27}$ miR-597 promoted cellular EMT to enhance the migration and invasion of NPC cells by targeting $14-3-3 \sigma$ (SFN). ${ }^{28}$ In this study, we found that the ectopic expression of miR379-5p obviously suppressed the EMT program, thereby inhibiting the migration and invasion of NPC cells, whereas the inhibition of miR-379-5p exerted the opposite effect. These data implicate that miR-379-5p is an important regulator of EMT in NPC.

MiRNAs regulate the EMT program of tumor cells mainly through repressing the expression of downstream EMT-related genes. Previously, Chen et al showed that miR-379-5p inhibited the EMT of HCC cells by targeting FAK expression to activate AKT signaling axis. ${ }^{11}$ Also, a study from $\mathrm{Xu}$ et al found that miR-379-5p/FAK axis played an important role in regulating the EMT of gastric cancer cells. ${ }^{13}$ In this study, we first indicated that miR-379-5p could suppress the EMT of NPC cells by regulating YBX1 expression to reduce cell migrative and invasive abilities. YBX1, as a multifunctional protein, is encoded by the YBX1 gene and can bind to the Y-box elements of many genes specifically. ${ }^{15}$ YBX1 plays different roles depending on the distribution location in cells (nucleus or cytoplasm). When located in the nucleus, YBX1 acts as a transcription regulator which regulates the expression of several genes by binding to the Y-box sequence located in the promoter contributing to transcription regulation, whereas YBX1 binds to mRNA to regulate translation process in the cytoplasm. ${ }^{16}$ Noteworthily, YBX1 was reported to activate Snail mRNA translation and induce the EMT in breast cancer. ${ }^{29}$ Therefore, YBX1 may play an important role in cells' EMT. ${ }^{30}$ Recently, YBX1 has been demonstrated to act as a tumor oncogene in the development and progression of human cancer, ${ }^{17,18}$ including NPC. ${ }^{20,21}$ Zhou et al found that YBX1 was upregulated in NPC tissues and its high expression positively correlated with $\mathrm{T}$ stage and metastasis of NPC patients, which could promote the EMT to increase NPC cells' migration and invasion. ${ }^{21}$ In addition, Zhang et al showed that LINC01133 inhibited EMT of NPC cells to suppress cell malignant-biological behavior by binding to YBX1. ${ }^{20}$ Combined with previous and our present studies, it is reasonably speculated that $\mathrm{YBX} 1$ promotes the oncogenesis and progression of NPC via promoting EMT-mediated tumor cells' malignant biological behaviors.

\section{Conclusions}

In summary, our study showed that the expression of miR$379-5 p$ is significantly decreased in NPC tissues, and its low expression is significantly associated with multiple unfavorable clinicopathological factors and poor prognosis of NPC patients. Mechanistically, miR-379-5p inhibits the cellular EMT by suppressing YBX1 to reduce the migration and invasion of NPC cells. These findings highlight the important role of miR-379-5p/YBX1 axis in suppressing EMT-mediated progression of NPC, providing a novel potential treatment target for NPC.

\section{Disclosure}

The authors report no conflicts of interest in this work.

\section{References}

1. Bray F, Ferlay J, Soerjomataram I, Siegel RL, Torre LA, Jemal A. Global cancer statistics 2018: GLOBOCAN estimates of incidence and mortality worldwide for 36 cancers in 185 countries. CA Cancer J Clin. 2018;68(6):394-424. doi:10.3322/caac.21492

2. Chen YP, Chan ATC, Le QT, Blanchard P, Sun Y, Ma J. Nasopharyngeal carcinoma. Lancet. 2019;394(10192):64-80. doi:10.1016/S0140-6736(19)30956-0

3. Hong X, Liu N, Liang Y, et al. Circular RNA CRIM1 functions as a ceRNA to promote nasopharyngeal carcinoma metastasis and docetaxel chemoresistance through upregulating FOXQ1. Mol Cancer. 2020;19(1):33. doi:10.1186/s12943-020-01149-x

4. Luo M, Wu C, Guo E, et al. FOXO3a knockdown promotes radioresistance in nasopharyngeal carcinoma by inducing epithelial-mesenchymal transition and the Wnt/beta-catenin signaling pathway. Cancer Lett. 2019;455:26-35. doi:10.1016/j.canlet.2019.04.019

5. Yang Z, Wang J, Zhang Z, Tang F. Epstein-barr virus-encoded products promote circulating tumor cell generation: a novel mechanism of nasopharyngeal carcinoma metastasis. Onco Targets Ther. 2019;12:11793-11804.

6. Brabletz T, Kalluri R, Nieto MA, Weinberg RA. EMT in cancer. Nat Rev Cancer. 2018;18(2):128-134. doi:10.1038/nrc.2017.118

7. Nieto MA, Huang RY, Jackson RA, Thiery JP. Emt: 2016. Cell. 2016;166(1):21-45. doi:10.1016/j.cell.2016.06.028

8. Di Leva G, Garofalo M, Croce CM. MicroRNAs in cancer. Annu Rev Pathol. 2014;9(1):287-314. doi:10.1146/annurev-pathol-012513-104715

9. Mutlu M, Raza U, Saatci O, Eyupoglu E, Yurdusev E, Sahin O. miR200c: a versatile watchdog in cancer progression, EMT, and drug resistance. J Mol Med (Berl). 2016;94(6):629-644. doi:10.1007/ s00109-016-1420-5

10. Suzuki HI. MicroRNA control of TGF-beta signaling. Int J Mol Sci. 2018;19(7):7. doi:10.3390/ijms19071901

11. Chen JS, Li HS, Huang JQ, et al. MicroRNA-379-5p inhibits tumor invasion and metastasis by targeting FAK/AKT signaling in hepatocellular carcinoma. Cancer Lett. 2016;375(1):73-83. doi:10.1016/j. canlet.2016.02.043 
12. Wu D, Niu X, Tao J, et al. MicroRNA-379-5p plays a tumor-suppressive role in human bladder cancer growth and metastasis by directly targeting MDM2. Oncol Rep. 2017;37(6):35 02-3508. doi:10.3892/or.2017.5607

13. Xu M, Qin S, Cao F, Ding S, Li M. MicroRNA-379 inhibits metastasis and epithelial-mesenchymal transition via targeting FAK/AKT signaling in gastric cancer. Int $J$ Oncol. 2017;51(3):867-876. doi:10.3892/ijo.2017.4072

14. Shi X, Xiao X, Yuan N, Zhang S, Yuan F, Wang X. MicroRNA-379 suppresses cervical cancer cell proliferation and invasion by directly targeting V-crk Avian sarcoma virus CT10 oncogene homolog-like (CRKL). Oncol Res. 2018;26(7):987-996. doi:10.3727/0965040 17X15140534417184

15. Kohno K, Izumi H, Uchiumi T, Ashizuka M, Kuwano M. The pleiotropic functions of the Y-box-binding protein, YB-1. Bioessays. 2003;25(7):691-698. doi:10.1002/bies.10300

16. Eliseeva IA, Kim ER, Guryanov SG, Ovchinnikov LP, Lyabin DN. Y-box-binding protein 1 (YB-1) and its functions. Biochemistry (Mosc). 2011;76(13):1402-1433. doi:10.1134/ S0006297911130049

17. Kuwano M, Shibata T, Watari K, Ono M. Oncogenic Y-box binding protein-1 as an effective therapeutic target in drug-resistant cancer. Cancer Sci. 2019;110(5):1536-1543. doi:10.1111/cas.14006

18. Lasham A, Print CG, Woolley AG, Dunn SE, Braithwaite AW. YB-1: oncoprotein, prognostic marker and therapeutic target? Biochem J. 2013;449(1):11-23. doi:10.1042/BJ20121323

19. Kosnopfel C, Sinnberg T, Schittek B. Y-box binding protein 1-a prognostic marker and target in tumour therapy. Eur J Cell Biol. 2014;93(1-2):61-70. doi:10.1016/j.ejcb.2013.11.007

20. Zhang W, Du M, Wang T, et al. Long non-coding RNA LINC01133 mediates nasopharyngeal carcinoma tumorigenesis by binding to YBX1. Am J Cancer Res. 2019;9(4):779-790.

21. Zhou LL, Ni J, Feng WT, et al. High YBX1 expression indicates poor prognosis and promotes cell migration and invasion in nasopharyngeal carcinoma. Exp Cell Res. 2017;361(1):126-134. doi:10.1016/j. yexcr.2017.10.009
22. Wang S, Claret FX, Wu W. MicroRNAs as therapeutic targets in nasopharyngeal carcinoma. Front Oncol. 2019;9:756. doi:10.3389/ fonc. 2019.00756

23. Lee KT, Tan JK, Lam AK, Gan SY. MicroRNAs serving as potential biomarkers and therapeutic targets in nasopharyngeal carcinoma: a critical review. Crit Rev Oncol Hematol. 2016;103:1-9. doi:10.1016/j.critrevonc.2016.04.006

24. He S, Yang S, Niu M, et al. HMG-box transcription factor 1: a positive regulator of the G1/S transition through the Cyclin-CDKCDKI molecular network in nasopharyngeal carcinoma. Cell Death Dis. 2018;9(2):100. doi:10.1038/s41419-017-0175-4

25. Ji W, Diao YL, Qiu YR, Ge J, Cao XC, Yu Y. LINC00665 promotes breast cancer progression through regulation of the miR-379-5p/ LIN28B axis. Cell Death Dis. 2020;11(1):16. doi:10.1038/s41419019-2213-X

26. Ding Y, Pan Y, Liu S, Jiang F, Jiao J. Elevation of MiR-9-3p suppresses the epithelial-mesenchymal transition of nasopharyngeal carcinoma cells via down-regulating FN1, ITGB1 and ITGAV. Cancer Biol Ther. 2017;18(6):414-424. doi:10.1080/15384047.2017.1323585

27. Lin C, Zong J, Lin W, et al. EBV-miR-BART8-3p induces epithelial-mesenchymal transition and promotes metastasis of nasopharyngeal carcinoma cells through activating NF-kappaB and Erk1/ 2 pathways. J Exp Clin Cancer Res. 2018;37(1):283. doi:10.1186/ s13046-018-0953-6

28. Xie L, Jiang T, Cheng A, et al. MiR-597 targeting 14-3-3sigma enhances cellular invasion and EMT in nasopharyngeal carcinoma cells. Curr Mol Pharmacol. 2019;12(2):105-114. doi:10.2174/ 1874467212666181218113930

29. Evdokimova V, Tognon $\mathrm{C}, \mathrm{Ng} \mathrm{T}$, et al. Translational activation of snail1 and other developmentally regulated transcription factors by YB-1 promotes an epithelial-mesenchymal transition. Cancer Cell. 2009;15(5):402-415. doi:10.1016/j.ccr.2009.03.017

30. Evdokimova V, Tognon C, Ng T, Sorensen PH. Reduced proliferation and enhanced migration: two sides of the same coin? Molecular mechanisms of metastatic progression by YB-1. Cell Cycle. 2009;8 (18):2901-2906. doi:10.4161/cc.8.18.9537

\section{Publish your work in this journal}

Cancer Management and Research is an international, peer-reviewed open access journal focusing on cancer research and the optimal use of preventative and integrated treatment interventions to achieve improved outcomes, enhanced survival and quality of life for the cancer patient.
The manuscript management system is completely online and includes a very quick and fair peer-review system, which is all easy to use. Visit http://www.dovepress.com/testimonials.php to read real quotes from published authors. 